inducing this gene expression program and in maintaining these macrophages at this location, which helped ameliorate inflammation. Functionally, activation of GATA6 by retinoic acid in these macrophages regulated the production of IgA by peritoneal B-1 immune cells.

Looking at other downstream effects of GATA6, Marcela Rosas and her colleagues found that mice lacking Gata6 had altered proliferation of tissue macrophages during homeostasis. In a mouse model of acute peritonitis, the absence of this factor inhibited recovery of macrophages and resulted in delayed resolution of inflammation. Although the authors did not pinpoint what induces GATA6 in peritoneal macrophages, ex vivo cultures confirmed that a local tissue signal was needed. $-C P$

\section{CARDIAC HYPERTROPHY}

\section{A miRNA star in the heart}

MicroRNAs (miRNAs) packaged in microvesicles or exosomes can help cells communicate with one another. Claudia Bang and her colleagues now show that this type of cell-cell interaction occurs between cardiac fibroblasts and muscle cells in culture and may promote pathological heart growth in mice (J. Clin. Invest. 124, 2136-2146, 2014).

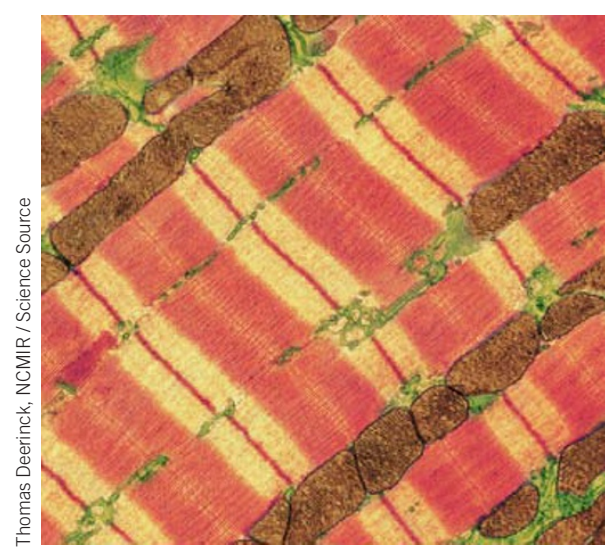

After showing that cultured cardiac fibroblasts release miRNA-containing exosomes, the authors found that one of these miRNAs, miR-21*, was transferred specifically into cultured cardiac muscle cells, causing an increase in their size. The authors then identified the gene encoding SORBS2 as a direct miR-21* target in the muscle cells. SORBS2 and another protein, PDLIM5, are good candidates for mediating the effect of miR- 21 * on cardiac muscle cell size, as deficiency of either protein led to increased cell size. In mice with pathological heart growth, the amounts of $\mathrm{miR}-21$ * in pericardial heart fluid were increased, and intravenous administration of a miR-21* antagomir reduced heart growth.

Although the 'passenger' or 'star' strand of miRNAs precursors is often degraded, the finding that cardiac fibroblast-derived exosomes were enriched in star strands-including $\mathrm{miR}-21$ * - points to the possibility that star strands might be preferentially involved in exosome-mediated communication.- $M B$

\section{NEURONAL DEVELOPMENT \\ Environmental brain protection}

The developing fetal brain is exposed to many environmental factors, some of which increase the risk of developing a psychiatric or neurological disease later in life. Now, Kazue Hashimoto-Torii and her colleagues report that maternal exposure to some environmental factors activates the stressresponsive transcription factor heat shock factor 1 (HSF1) in fetal neurons in mice and humans, which seems to protect the brain from these insults (Neuron 82, 560-572, 2014).

The researchers showed that maternal exposure to alcohol, methylmercury or chemically induced seizures could increase binding of HSF1 to one of its response genes in the brains of fetal mice. This binding was also enhanced in human fetal brain slices exposed to alcohol. In the absence of HSF1 in fetal mice, normally benign amounts of these environmental factors promoted abnormal neuronal positioning, a reduction in the thickness of the cortex, increased cell death of neural progenitor cells and a brain more prone to seizures.

In neural progenitor cells that were derived from human induced pluripotent stem cells, the authors found that the extent to which these environmental factors could induce HSF1 signaling was more variable in cells from patients with schizophrenia compared with controls. The findings suggest that variable activation of a stress response system to environmental factors in developing neurons could play a role in enhancing disease risk in these patients. $-E C$

\section{DENGUE VIRUS}

\section{An enhanced model for dengue virus}

Upon initial infection with any of the four serotypes of dengue virus (DENV), an acute

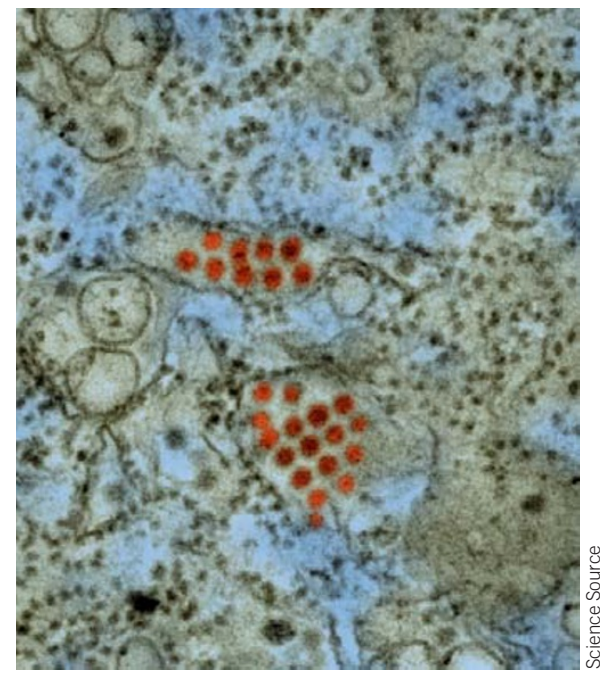

flu-like illness ensues; however, subsequent infection with a different subtype can cause a potentially lethal hemorrhagic fever. Despite the high incidence of DENV infection, there is no approved vaccine and only limited animal models to mimic the antibody-dependent enhancement (ADE) of infection that is thought to be responsible for the lethal dengue fever. A new mouse model of ADE associated with maternal antibodies may help explain the increased susceptibility of some infants to severe dengue fever ( $P$ LoS Pathog. 10, e1004031, 2014).

Jowin Kai Wei Ng and colleagues infected female mice with a clinical isolate of DENV serotype 1 (DENV1) and infected offspring from these mothers with a different serotype, DENV2, to induce lethal disease. The offspring of mothers that developed antibodies against DENV1 showed more rapid onset of disease and death at 5 weeks of age compared to DENV2-infected offspring of naive mothers. The age at which offspring were infected with the second DENV serotype after birth also influenced the course of the disease.

The findings suggest that although high maternal antibody levels against DENV1 in newborn mice can cross-neutralize DENV2 and slow disease progression, they wane with aging of the offspring, and subneutralizing concentrations may no longer block infection. Instead, these lower amounts of antibodies can mediate uptake of antibodybound virus by monocytes, and facilitate increased viral proliferation in these cells, leading to enhanced disease.-AF

Written by Michael Basson, Eva Chmielnicki, Alison Farrell, Randy Levinson, Carolina Pola, Kendra Simpson \& Hannah Stower 